\title{
Knowledge, usefulness and use of checklists in surgical areas in a private hospital, running title: usefulness of checklists in surgical areas
}

\begin{abstract}
Background: Errors in medicine have serious consequences, for this reason efficient prevention strategies such as checklists are necessary. Despite its benefits, sometimes surgical staff ignores importance of checklists or is reluctant to apply them. The objective was to investigate the knowledge surgical personnel has about the impact and correct implementation of this protocol.
\end{abstract}

Methodology: Prospective, observational, descriptive study. Surveys were carried out to the surgical personnel of Hospital Angeles Lomas evaluating their knowledge about the checklists. The Microsoft Excel ${ }^{\circledR}$ V .12.3.6 and SPSS v.21 programs were used for data collection and analysis. Student's t-test and Pearson's correlation were used looking for significance and correlation between application of the protocol, communication of the team, impact on morbidity and mortality and the attitude of surveyed people about checklists. We investigate also the most frequent omissions when applying the protocol.

Results: Of 75 surveys, 69 were suitable to analysis, more than half of the people knew the protocol and recognized its impact; surgeons and nurses recognized their practicability. $46.3 \%$ didn't know the phases and the correct time to carry out the protocol. The most perceived omissions were: surgical marking, equipment presentation and verification of prevention of deep venous thrombosis.

Conclusions: Checklists are a simple and economical tool to improve security, morbidity and mortality rates in surgical patients. However, there are still barriers to its correct use and implementation. Staff understanding and training is required to acquire this routine in daily practice and implement it correctly.

Keywords: Checklists, patient security, surgical time-out, medical errors, morbidity, mortality, anesthesiologists, improve security, venous thrombosis, surgical marking, gauzes, textiles, surgical personnel, nurses

Volume 6 Issue 5 - 2018

\author{
Alejandro Weber-Sánchez , Pablo Weber- \\ Álvarez, Dra.Ana Sofía Mateos-López, Mariel \\ de la Jara, Rita Cohen-Cohen \\ Department of surgery, Hospital Ángeles Lomas, México
}

\section{Correspondence: Alejandro Weber Sánchez,Vialidad de la Barranca s/n C4I0,Valle de las Palmas, Huixquilucan, 52763, Estado de México, México. Tel: 52469527}

Email awebersanchez@gmail.com

Received: July 2, 20I8| Published: September I2, 2018

\section{Introduction}

The human being is fallible by nature, however many of the committed mistakes can be avoided, and prevention in many cases is within our control. In medicine, especially surgery, given the complexity of the processes and the number of participants in them, errors despite knowledge and experience even in routine procedures are frequent and potentially have serious consequences. ${ }^{1}$ Therefore, it is necessary to develop effective prevention and control strategies since the life and safety of the patients could be at risk. One of the strategies that have proven effective reduction of morbidity and mortality in the operating room is the use of checklists. Although their effectiveness and impact on the reduction of morbidity and mortality in surgery has been demonstrated since many years, correct implementation of this protocol has not been easy due to different factors, mainly the lack of knowledge of their function and impact, which causes failure in their implementation and reluctance to change. The objective of this investigation was to determine the knowledge of surgical personnel about the impact and correct implementation of this procedure in a general private hospital in Mexico before its full implementation.

\section{Methodology}

This is a prospective, observational, descriptive study, through the application of a questionnaire to the surgical staff of the Hospital Angeles Lomas in Huixquilucan, State of Mexico, in relation to the preoperative safety protocol known as "time out", with the objective to evaluate the knowledge and attitude towards checklist in the surgical area. The survey was designed using the judging system with members of the hospital's quality committee and head of general surgery department and nurse staff. We included 15 questions of different type including questions about knowledge about this process, its usefulness and impact on the decrease of morbidity and mortality of surgical patients, attitude to the process and specific points of how it is performed. The questionnaire was previously piloted with a group of 10 surgeons to verify their understanding of the questions and based on the result; those that generated confusion were modified to have the final version. The questionnaire was applied to surgeons of different surgical specialties, mainly general surgery, surgical nurses and surgical residents who voluntarily agreed to answer the questionnaire. The questionnaires that were not completed adequately were eliminated. The investigation was approved by the hospital ethics committee. For the collection and coding of the database, the Microsoft Excel ${ }^{\circledR}$ v. 12.3.6 program was used. The SPSS v. 21 programs was used to perform the descriptive and analytical statistics. The normality of the surveyed population was verified by the Kolmogorov-Smirnoff test. Student's t test was used to find statistical significance between the different variables and questions, and the Pearson correlation coefficient to show if there was a correlation between the application of the protocol, team communication and the impact on morbidity and mortality related to the profession and the age group, considering statistically significant the value of $\mathrm{p}<0.05$. 


\section{Results}

75 questionnaires were applied, 6 of which were eliminated due to lack of adequate filling. Of the 69 useful questionnaires, 27 (39.1\%), corresponded to the age group between 20 to 30 years, $12(17.3 \%)$ between 31 to $40,19(27.5 \%)$ from 41 to 50 and 11 (15.9\%) belonged to the group of over 50 years old (Figure 1). According to their profession $26(37.6 \%)$ were surgeons, $5(7.2 \%)$ anesthesiologists, $27(39.1 \%)$ nurses and $11(15.9 \%)$ belonged to another branch of the surgical staff.

\section{AGE GROUP}

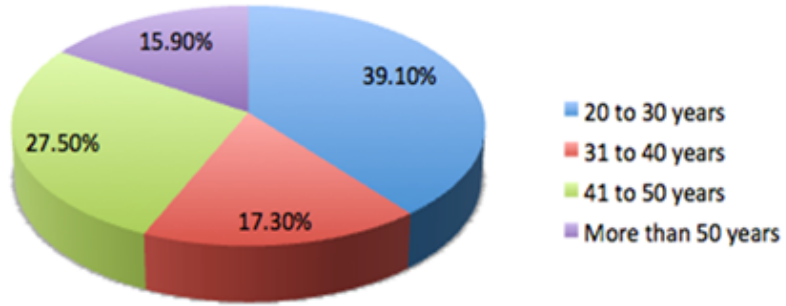

Figure I Age Group.

Analyzing the answers, in terms of knowledge of the patient's safety protocol, 35 (50.7\%) people answered that they knew it very well (16 nurses, 14 surgeons, 2 anesthesiologists, 3 others), 25 (36.2\%) were familiar with it ( 8 surgeons, 10 nurses, 3 anesthesiologists, 4 others), 7 $(10.1 \%)$ had heard about it (3 surgeons, 4 others) and $1(1.4 \%)$ did not know about it (1 surgeon) (Figure 2). Considering the practicability of its application, 67 (97.1\%) of the respondents considered it practical, and $2(2.8 \%)$ did not consider it as such (Figure 3). Regarding the impact about the communication of the surgical team, $60(86.9 \%)$ answered that it has an impact and $9(13 \%)$ not. In relation to the impact of checklists to reduce morbidity and mortality, $61(88.4 \%)$ answered that it has a positive impact and $8(11.5 \%)$ not. (Figure 4$)$. Regarding the attitude of the respondents with respect to the protocol, $31(44.9 \%)$ replied that it should be collaborative, $31(44.9 \%)$ of acceptance (12 surgeons, 2 anesthesiologists, 13 nurses, 4 others) and $7(10.1 \%)$ of enthusiasm. When evaluating the knowledge about the protocol phases, $37(53.6 \%)$ participants answered correctly (21 surgeons, 3 anesthesiologists, 8 nurses, 5 others) and 32 (46.3\%) not. Regarding the marking of the surgical site according to the protocol established by the institution, $53(76.8 \%)$ people had knowledge about the mark used and $16(23.1 \%)$ did not, in relation to the cases in which the surgical marking applied $57(82.6 \%)$ people answered adequately and $12(17.3 \%)$ did not. $65(94.2 \%)$ people answered adequately who is responsible for the surgical marking and $4(5.7 \%)$ not. According to the moment the safety protocol must be carried out, $44(63.7 \%)$ answered adequately and $25(36.2 \%)$ did not. As for the moment when the time out should be done, 38 (55\%) people knew it and $31(44.9 \%)$ not. In relation to the knowledge of the ideal time to administer antibiotic prophylaxis $45(65.2 \%)$ answered adequately and $24(34.7 \%)$ did not.

To evaluate the most frequent omissions in the verification procedure, the surveyed population were asked to mark all the options they considered people omitted when applying the protocol from the list provided, $8(11.5 \%)$ answered that the most frequent omission was the identification of the patient, $13(18.8 \%)$ the verbal confirmation of the surgical site, $8(11.5 \%)$ verification of the procedure to be performed, $17(24.6 \%)$ omission of the surgical marking, $15(21.7 \%)$ omission of anesthesia equipment check, $15(21.7 \%)$ the allergic status of the patient, $39(56.5 \%)$ presentation of the surgical team members, $30(43.4 \%)$ verification of the deep venous thrombosis prevention and $17(24.6 \%)$ verification of the antibiotic prophylaxis. Regarding the identification of the phase in which the final count of the gauzes and textiles should be confirmed, $62(89.8 \%)$ answered adequately and 7 $(10.1 \%)$ did not. When applying the Pearson coefficient, correlation was found regarding the profession of the respondents and the perception about the impact on morbidity and mortality (p 0.036). Using the student's t-test for related samples, none of the professions of the surveyed personnel showed statistical significance regarding their perception of the impact on morbidity and mortality (surgeon p 0.80 , anesthesiologist $\mathrm{p} 0.057$, nurse 0.082 , others $\mathrm{p} 0.061$ ). On the contrary, people of all professions showed statistical significance regarding their attitude to the protocol ( $\mathrm{p} 0.000)$. When considering group of age and their attitude we found the older group had better attitude ( $\mathrm{p} 0.000)$ than the youngest ( $\mathrm{p} 0.112)$.

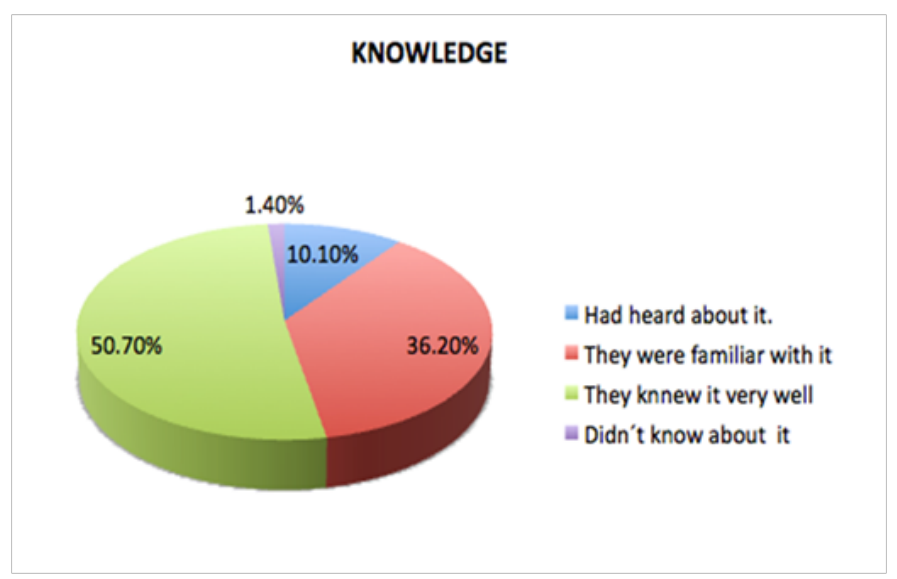

Figure $\mathbf{2}$ Knowledge of the safety protocol.

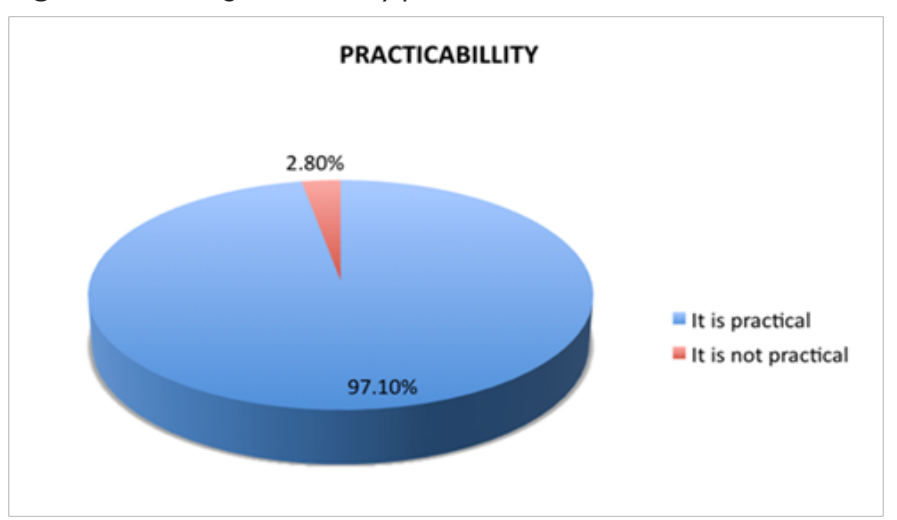

Figure 3 Practicabillity of the safety protocol.

\section{Discussion}

The checklists have been implemented as a useful and simple tool to increase safety and reduce morbidity and mortality in surgical practice, and has a favorable impact on hospitals that apply this protocol throughout the world, in both developed and middle-income countries $^{2}$, being these kind of countries those that have been able 
to reduce up to 6 times the risk of failure at critical moments of surgical management using these lists ${ }^{3}$. The level of knowledge and application of these protocols is diverse in different institutes, and concerns not only surgeons but also all the staff that works in the operating room. For example, Hurtado et $\mathrm{al}^{4}$. in Guatemala, found that $93 \%$ of the surgical staff knew of its existence and $88.8 \%$ were aware of its objectives, having the nurses the best acceptance of the protocol and also being those who showed the best training for its application ${ }^{5}$. Our study also showed that nurses and surgeons were familiar with the protocol and its implementation, and that it has a positive impact in the patient's safety in surgery, as Gawande wrote in his book "The

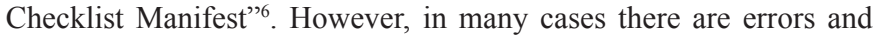
deficiencies in its correct application.

\section{IMPACT ON MORBIDITY AND MORTALITY}

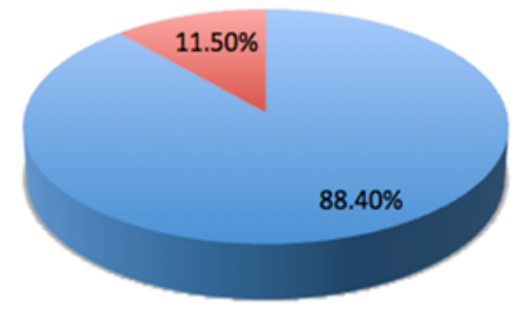

\section{It has a positive impact = It does not has a positive impact}

Figure 4 Impact on morbidity and mortality.

Despite the benefits observed when implementing the checklists it has been demonstrated that the level of staff attachment to it, is related to its effectiveness, ${ }^{7}$ increasing the perception of teamwork quality, communication improvement and error reduction. In addition to this, it is considered a quality instrument in hospitals. Russ et al. evaluated surgeries in real time, finding that safety measures were performed in less than one third of all procedures and Poon et al ${ }^{8-10}$ evaluated 193 procedures of which only $78.4 \%$ fulfilled completely the 11 elements of the time out, and found that the most omitted elements were: verification of the presence of the complete surgical team, corroboration of the patient's identity, the surgical site, allergies and preoperative discussion of special considerations, all of which evidenced the need of creating consciousness and improve staff training to the fulfillment and adequate implementation of this protocol. Our study showed similar results in terms of the respondents assessment that it is in many cases incompletely performed, missing important points such as the verification of anti-thrombotic prophylaxis which almost half of the respondents believe is omitted, and a quarter of them think that the verification of the timely application of antibiotic prophylaxis and surgical marking is omitted, considered as fundamental elements that have a special influence on the patient's safety ${ }^{10,14}$ and other important lapses of the protocol.

Another common problem in its application is the lack of knowledge regarding its phases. Almost half of the respondents did not answer correctly the moment when the time out should be executed. And more than a third did not answer correctly the optimal time for the application of the prophylactic antibiotic. A factor that contributes to this phenomenon is that in many cases the superiors do not make enough awareness among the subordinates about the reasons for its application, causing frustration, disinterest and abandonment. ${ }^{12,13}$ Different barriers have been identified for the effective implementation of checklists such as poor communication among physicians, ambiguity about the reasons for their application, ignorance of the avoidable risks and problems with the authority. ${ }^{8,15,16}$

As it can be seen, in our study and other similar studies, the attitude of the respondents in general is of acceptance and collaboration, however, due to omissions and lack of knowledge regarding the correct application, it seems that in many cases the real impact in the reduction of morbidity and mortality of surgical patients is not taken into account, or that it is taken as a "formality" that increases the workload and consumes valuable time. ${ }^{17}$ For this reason it is important that the surgical staff is persuaded of its importance and its implementation, promoting a culture of safeness. ${ }^{18}$ In general, there is good attitude as this research shows, but better results can be achieved through strategies such as motivational conferences and intensive courses to raise awareness and achieve its correct application by all surgical personnel $\mathrm{l}^{19,20}$ as well as continuous monitoring and evaluation to improve the effectiveness and safety of surgical patients.

\section{Conclusions}

It has been shown that checklists are a simple and economic tool that improves morbidity and mortality rates in surgical patients. However, there are still many barriers to its correct use and implementation. Training and awareness of the staff is required so that they can have a deeper knowledge and it can be applied properly in daily practice.

\section{Acknowledgement}

None.

\section{Conflict of Interest}

Authors declare that there is no conflict of interest.

\section{Reference}

1. Gorovitz S, Maclntyre A. Toward a Theory of Medical Fallibility. J Med Philos. 1976;1(1):51-71.

2. Jain D, Sharma R, Reddy S. WHO safe surgery checklist: Barriers to universal acceptance. J Anaesthesiol Clin Pharmacol. 2018;34(1):7-10.

3. Ziewacz J, Arriaga A, Bader A, et al. Crisis Checklists for the Operating Room: Development and Pilot Testing. J Am Coll Surg. 2011;213(2):212217.

4. Hurtado DJ, Jimenez X, Penolonzo M. Acceptance of the WHO Surgical Safety Checklist among surgical personnel in hospitals in Guatemala city. BMC Health Services Research. 2012;169:2-5.

5. Wahle VH, Haugen SA. Adjusting team involvement: a grounded theory study of challenges in utilizing a surgical safety checklist as experienced by nurses in the operating room. BMC Health Serv Res. 2012;16:2-10.

6. Gawande A. The Checklist Manifesto: How to get things wright. Nueva York: Picador; 2010;3(2):124.

7. Borchard A, Schwappach DL, Barbir A, et al. A systematic review of the effectiveness, compliance, and critical factors for implementation of safety checklists in surgery. Ann Surg. 2012;256(6):925-33.

8. Russ S, Rout S, Sevdalis N, et al. Do Safety Checklists Improve Teamwork and Communication in the Operating Room? A Systematic Review. Ann Surg. 2013;258(6):856-71.

9. Poon SJ, Zuckerman SL, Mainthia R, et al. Methodology and bias in assessing compliance with a surgical safety checklist. Jt Comm J Qual Patient Saf. 2013;39(2):77-82. 
10. van Schoten MS, Kop V, de Blok C, et al. Compliance with a time-out procedure intended to prevent wrong surgery in hospitals: results of a national patient safety programme in the Netherlands. BMJ Open. 2014;4(7):e005075.

11. Malhotra MK, Malhotra S, Chowdhary K, et al. Surgical Safety Checklist Popularity among the surgeons?A survey. Bangladesh Journal of Medical Science. 2017;16(4):521-524.

12. Conley D, Singer S, Edmondson L. Effective Surgical Safety Checklist Implementation. J Am Coll Surg. 2011;212(5):873-79.

13. Haugen SA, Murugesh S, Haaverstad R, et al. A survey of surgical team members' perceptions of near misses and attitudes towards Time Out protocols. BMC Surg. 2013;13-46.

14. Gibbs V. Thinking in three's: Changing surgical patient safety practices in the complex modern operating room. World $J$ Gastroenterol. 2012;18(46):6712-19.
15. Fourcade A, Blache J, Grenier C, et al. Barriers to staff adoption of a surgical safety checklist. BMJ Qual Saf. 2012;21(3):191-97.

16. Aledo SV, Da Silva ZA, Saturno P, et al. Dificultades en la implantación del check list en los quirófanos de cirugía. Cirugía Española. 2012;90(3):180-185.

17. Stock C, Sundt T. Timeout for Checklists?. Ann Surg. 2015;261(5):841842 .

18. Norra MacReady. OR Briefings Reduce Surgical Errors, Improve Outcomes. 2014; Available at: https://www.medscape.com/ viewarticle/828127. Accessed 06/07, 2018.

19. Warnock LG. Of surgeons and safety checklists. Can J Surg. 2010;56(6):364-365.

20. White MC, Baxter LS, Close KL, et al. Evaluation of a countrywide implementation of the world health organisation surgical safety checklist in Madagascar. PLoS One. 2018;13(2): e0191849. 